\title{
Research Paper: Dynamic Balance During Gait in Children With Spastic Diplegic Cerebral Palsy Ver- sus Normal Children
}

\author{
Keyvan Sharifmoradi $^{1}$ (D, Mostafa Kamali ${ }^{*}$ (D), Ali Tahmasebi ${ }^{3}$ (D)
}

1. Department of Physical Education and Sport Sciences, Faculty of Humanities, University of Kashan, Kashan, Iran. 2. Department of Physiotherapy, School of Rehabilitation Sciences, Isfahan University of Medical Sciences, Isfahan, Iran. 3. Department of Occupational Therapy, School of Rehabilitation, Tehran University of Medical Sciences, Tehran, Iran.

\begin{tabular}{|c|c|}
\hline $\begin{array}{l}\text { Use your device to scan } \\
\text { and read the article online }\end{array}$ & $\begin{array}{l}\text { Palsy Versus Normal Children. Physical Treatments. 2018; 8(1):9-16. http://dx.doi.org/10.32598/ptj.8.1.9 } \\
\text { doi: : http://dx.doi.org/10.32598/ptj.8.1.9 }\end{array}$ \\
\hline
\end{tabular}

\section{(i) (3)}

\section{Article info:}

Received: 14 Dec 2017

Accepted: 28 Feb 2018

Available Online: 01 Apr 2018
Keywords:

Center of mass, Base of support, Dynamic stability, Cerebral Palsy

\begin{abstract}
A B S T R A C T
Purpose: Cerebral Palsy (CP) can negatively affect dynamic stability in children with spastic diplegic Cerebral Palsy during walking. This condition results in a high risk of falling. There is limited evidence regarding the dynamic stability of children with Cerebral Palsy. Thus, this study aimed to investigate the dynamic stability of children with spastic diplegic Cerebral Palsy, compared to typically developed children during walking.
\end{abstract}

Methods: Sixteen children including 8 with spastic diplegic Cerebral Palsy and 8 normal ones with an age range of 5 to 8 years participated in this quasi-experimental study. A Qualysis motion analysis system capturing at a frequency of $100 \mathrm{~Hz}$ was used to record data. Qualysis software and Visual3D software were utilized for data extraction. Data analysis was conducted using the Independent t-test with $\mathrm{P}<0.05$.

Results: The stride length and velocity of gait in children with Cerebral Palsy were $32.25 \mathrm{~cm}$ and $0.34 \mathrm{~m} / \mathrm{s}$ lower than the normal children, respectively. Center of mass displacement was $12.25 \%$ lower in anteroposterior plane and $1.23 \%$ higher in mediolateral plane in children with Cerebral Palsy, compared to the normal children. The margin of stability was $1.72 \mathrm{~cm}$ higher in the children with Cerebral Palsy, compared to normal children.

Conclusion: The lower anteroposterior and higher mediolateral displacement of the center of mass result in an altered pattern of gait in children with Cerebral Palsy, compared to the normal children. Considering the aforementioned changes and the lower velocity of gait in children with Cerebral Palsy, the dynamic stability of children with spastic diplegic Cerebral Palsy is lower, compared to the normal children during walking.

\footnotetext{
* Corresponding Author:

Mostafa Kamali, MSc.

Address: Department of Occupational Therapy, School of Rehabilitation, Tehran University of Medical Science, Tehran, Iran.

Phone: +98 (913) 3505228

E-mail:mostafa_kamali@rehab.mui.ac.ir
} 


\section{Highlights}

- Center of mass is lower in anteroposterior and higher in mediolateral direction in children with CP compared to normal children.

- Margin of stability increases in children with CP compared to normal children.

- Dynamic stability of CP children is lower.

\section{Plain Language Summary}

Cerebral palsy (CP) can negatively affect dynamic stability in children with spastic diplegia CP during walking. This condition results in a high risk of falling. So, this study aims to investigate the dynamic stability of children with spastic diplegia $\mathrm{CP}$ and compare it with typically-developed children during walking. CP children walk with lower stride length and speed compared to normal children. Center of mass displacement was also lower in CP children but side to side displacement of center of mass was wider compared to normal children. The margin of stability was higher in the children with CP, thus the dynamic stability of these children was lower compared to normal children during walking. So to increase the dynamic stability of CP children during walking, such exercises are useful that reduce the step width and increase the stride length and walking speed.

\section{Introduction}

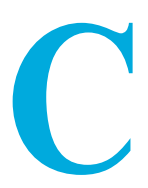

erebral Palsy (CP) is a neurological disorder caused by damage or lack of brain maturation. Although brain damage is not progressive, some abnormalities occur in the musculoskeletal system of children which cause reduced stride length and increased double support time $[1,2]$. It seems that these changes lead to an unstable walking pattern. The postural balance in children with $\mathrm{CP}$ is weak and puts them at risk of falling [2]. However, few studies have been conducted on the dynamic balance of walking in children with CP [3-7].

Balance is the ability of a person to keep the body in a stable position and return from an unstable to a stable condition $[8,9]$. It also includes balance in an upright standing position and dynamic balance (while performing various tasks like walking). There are some studies on upright standing balance of children with CP $[10,11]$. These studies investigated upright standing balance in different positions like tandem and normal stances or in variable sensory conditions like standing with eyes open and closed [12-14].

Standing balance test results cannot be generalized to walking balance $[14,15]$, and fail to predict the risk of falling during walking $[16,17]$. This is because walking is an intrinsically unstable activity where the Center of Mass (COM) is constantly fluctuating uniformly, and may be transferred to the outside of Base of Support (BOS) during single support $[5,18]$. Therefore, we can predict the fall risk only by assessing the balance during walking $[17,19]$.

Children with CP show a different walking pattern than the normal children. The gait speed in these children is lower to increase their stability $[4,7]$. They increase the step width to maintain their lateral balance [4]. They also reduce the step length to increase the balance in posterior/anterior directions. The increased step width observed in those with less balance [20], and in CP children, is accompanied by an increase in energy expenditure [21]. This walking pattern caused by lack of dynamic balance increases the risk of falling in CP children. Therefore, the dynamic balance of patients is of great importance. Improving the dynamic balance of children with $\mathrm{CP}$, depends on the precise identification of the dynamic balance weaknesses.

Few studies are available on the dynamic balance of CP children. Some drawbacks of these studies include the lack of upper body markers [3], invalidity of models to assess children's balance [3], lack of Margin of Stability (MOS) calculations [7], use of safety belts during walking [4], variable age range [3, 4, 6, 7], and variable intensity of CP. We assessed the dynamic balance of children with spastic diplegic $\mathrm{CP}$ at a low age range; also we calculated the MOS in these children during walking. 


\section{Materials and Methods}

In this causal-comparative study, 8 children with $\mathrm{CP}$ participated with the Mean \pm SD age, height and weight of $7 \pm 1.3$ years, $106.25 \pm 5.11 \mathrm{~cm}$, and $17.23 \pm 2.86 \mathrm{~kg}$, respectively. They were recruited from $\mathrm{CP}$ children referring to Neurology centers in Isfahan City, Iran, using convenience sampling method. Inclusion criteria were having spastic diplegic $\mathrm{CP}$, ability to stand and walk independently, no use of walker or other assistive devices, no history of lower limbs and trunk surgery affecting walking, ability to follow the verbal instructions, no history of treatment with botulinum toxin within the last 6 months [3], and being categorized at level II or III based on Gross Motor Function Classification System (GMFCS). An informed consent form was obtained from the parents of the children before conducting any measurement. Eight normal children were also randomly selected and participated in this study (control group).

The kinematic data was collected at $100 \mathrm{~Hz}$ using a 7-camera motion capture system (Qualisys, Qualisys Company, Switzerland). Twenty-two 14-mm diameter illuminated markers were attached to the bony landmarks of the subjects using infrared lights, according to the protocol approved by the University of Strathclyde. The cameras could detect the position of the markers. At first, a static test was performed on the subjects followed by dynamic tests. The length of the walking path inside the laboratory was $10 \mathrm{~m}$, where the distance between starting point and destination was $5 \mathrm{~m}$.

A calibration space $($ length $\times$ width $\times$ height $=200 \times 100 \times 300$ $\mathrm{cm}$ ) was defined in the laboratory environment and in the middle of the walkway. All data were recorded in this place. All stages of the test were performed by a gait lab expert from the School of Rehabilitation Sciences, Isfahan University of Medical Sciences. Before performing the main tests, each subject performed 2 gait trials in order to get acquainted with the method of testing. Then, the main tests were conducted. In this regard, 3 gait trials were performed on each subject and the mean value of 3 trials was used for subsequent calculations.

During the gait trial, the subjects were barefoot wearing only boxer underwear. The labeling of markers was based on the position of each marker in the software environment. Also, the registration of the static and dynamic phases of walking, and the measurement of spatial and temporal gait parameters were done using the Qualisys software (Qualisys, Switzerland). The Qualisys outputs were exported to Visual3D (version 4, C-motion Inc., USA) for modeling the subjects. The data were filtered at
$10 \mathrm{~Hz}$. Eventually, 9 segments (feet, shank, thighs, head, and trunk) were generated to model the patient's body. Finally, the coordinates of the COM were extrapolated to 3 motion screens during the full gait cycle.

The study variables consisted of stride time, step length, gait speed, cadence, raw and normalized motion ranges of COM in vertical, anterior/posterior and medial/lateral planes, and MOS. The MOS can be defined by subtracting the range of motion of extrapolated COM in medial/ lateral plane from the BOS during stance phase of each step [22]. The variables of the COM were normalized according to the leg length and pelvic width, to eliminate the effects of height on the results [23-26]. The leg length refers to the distance of marker attached to the hip from the ground per centimeter. The pelvic width is the transverse distance between the 2 anterior superior iliac spines on the right and left sides per centimeter. The BOS is the transverse distance between the fifth metatarsal marker on the right and left side, during stance phase of each step [27].

To test the normality of data distribution, ShapiroWilk test was employed. Data analysis was conducted in SPSS using Independent t-test. The significance level was set at $0.05(\mathrm{P}<0.05)$.

\section{Results}

Table 1 presents the demographic characteristics of participants in both groups of CP and control. The spatial and temporal gait parameters for both groups are listed in Table 2. No significant differences were observed between the 2 groups in terms of cadence and stride time. However, there was a significant difference between them in terms of step length and gait speed. The step length of CP children was $32.25 \mathrm{~cm}$ less than that of the controls $(\mathrm{P}<0.01)$. Moreover, in comparison with the control group, their gait speed was $0.34 \mathrm{~m} / \mathrm{s}$ slower $(\mathrm{P}<0.01)$.

According to Table 3 which presents dynamic balance variables during walking for both study groups, the non-normalized Mean \pm SD motion range of COM in $\mathrm{CP}$ children in vertical plane was $1.35 \pm 0.54 \mathrm{~cm}$, which is similar to the controls $(\mathrm{P}=0.10)$. In anterior/posterior plane, the Mean $\pm \mathrm{SD}$ range was $17.93 \pm 8.64 \mathrm{~cm}$ which is $15.16 \mathrm{~cm}$ less than the range reported for the controls $(\mathrm{P}<0.01)$. However, in medial/lateral plane, it was 6.47 (2.12) which is $2.41 \mathrm{~cm}$ more than that of the control group $(\mathrm{P}=0.02)$. Moreover, the normalized motion range of COM in $\mathrm{CP}$ children in vertical plane was similar to that of the controls $(\mathrm{P}=0.36)$. 
Table 1. Demographic characteristics of the study participants

\begin{tabular}{ccc}
\hline & \multicolumn{2}{c}{ Mean \pm SD } \\
\cline { 2 - 3 } & CP & Control \\
\hline Gender & 6 girls, 2 boys & 5 girls, 3 boys \\
\hline Age, $y$ & $7 \pm 1.3$ & $7.2 \pm 1.1$ \\
\hline Weight, $k g$ & $17.23 \pm 2.86$ & $23.29 \pm 7.15$ \\
\hline Height, $c m$ & $106.25 \pm 5.11$ & $124.63 \pm 10.59$ \\
\hline Leg length, $c m$ & $44.63 \pm 16.38$ & $58.53 \pm 5.52$ \\
\hline Pelvic width, $c m$ & $23.56 \pm 5.49$ & $25.01 \pm 4.59$ \\
\hline
\end{tabular}

PHYSICAL TREA $\$ MENTS

Table 2. Spatial and temporal gait parameters in the $\mathrm{CP}$ and control groups (right leg)

\begin{tabular}{|c|c|c|c|c|}
\hline \multirow{2}{*}{ Parameters } & \multicolumn{2}{|c|}{ Mean $\pm S D$} & \multirow{2}{*}{ 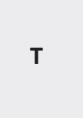 } & \multirow{2}{*}{ Sig. } \\
\hline & CP & Control & & \\
\hline Stride time, s & $1.21 \pm 0.26$ & $1.03 \pm 0.11$ & 1.75 & 0.10 \\
\hline Step length, cm & $39.5 \pm 18.81$ & $71.75 \pm 20.40$ & 3.28 & $0.005^{*}$ \\
\hline Cadence & $106.17 \pm 31.09$ & $117.68 \pm 12.01$ & 0.976 & 0.345 \\
\hline Gait speed, $\mathrm{m} / \mathrm{s}$ & $0.35 \pm 0.19$ & $0.69 \pm 0.19$ & 3.67 & 0.003 \\
\hline
\end{tabular}

* Significant

PHYSICAL TREA MENTS

They had significant differences with each other in anterior/posterior $(\mathrm{P}<0.01)$ and medial/lateral $(\mathrm{P}=0.02)$ planes. In anterior/posterior plane, the normalized motion range of COM in CP children was $12.25 \%$ lower and $1.23 \%$ higher in medial/lateral than the control group (Figure 1). Furthermore, the Mean \pm SD score of MOS in $\mathrm{CP}$ children was $3.43 \pm 1.80 \mathrm{~cm}$. This value was $1.72 \mathrm{~cm}$ higher than the MOS in the controls ( $\mathrm{P}=0.02)$ (Figure 2).

\section{Discussion}

We investigated the dynamic balance of children with $\mathrm{CP}$ in comparison with their norrs during walking. According to the results, the dynamic balance of these children in anterior and posterior directions was significantly lower compared to normal ones, and their MOS was higher.

Table 3. Dynamic balance variables during walking in $\mathrm{CP}$ and control groups

\begin{tabular}{|c|c|c|c|c|c|}
\hline \multirow{2}{*}{ Variable } & \multirow{2}{*}{ Direction } & \multicolumn{2}{|c|}{ Mean \pm SD } & \multirow{2}{*}{$\mathbf{T}$} & \multirow{2}{*}{ Sig. } \\
\hline & & $\mathrm{CP}$ & Control & & \\
\hline \multirow{3}{*}{$\begin{array}{l}\text { Motion range of COM } \\
\text { (non-normalized data) }\end{array}$} & Vertical & $1.35 \pm 0.54$ & $1.76 \pm 0.39$ & -1.74 & 0.10 \\
\hline & Anterior/posterior & $17.93 \pm 8.64$ & $33.77 \pm 9.64$ & -3.55 & $0.003^{*}$ \\
\hline & Medial/lateral & $6.47 \pm 2.12$ & $4.06 \pm 1.25$ & 2.77 & $0.02 *$ \\
\hline \multirow{3}{*}{$\begin{array}{l}\text { Motion range of COM } \\
\text { (normalized data) }\end{array}$} & Vertical to leg length (\%) & $2.64 \pm 0.95$ & $3 \pm 0.53$ & -0.94 & 0.36 \\
\hline & Anterior/posterior to leg length (\%) & $35 \pm 15.02$ & $57.25 \pm 13.49$ & 0.552 & $0.008^{*}$ \\
\hline & Medial/lateral to pelvic width & $2.88 \pm 1.22$ & $1.65 \pm 0.55$ & 2.62 & $0.02 *$ \\
\hline MOS & $\begin{array}{l}\text { Subtracting transverse motion } \\
\text { range of COM from the step width }\end{array}$ & $3.43 \pm 1.80$ & $1.71 \pm 0.59$ & 2.57 & $0.02 *$ \\
\hline
\end{tabular}

* Significant 


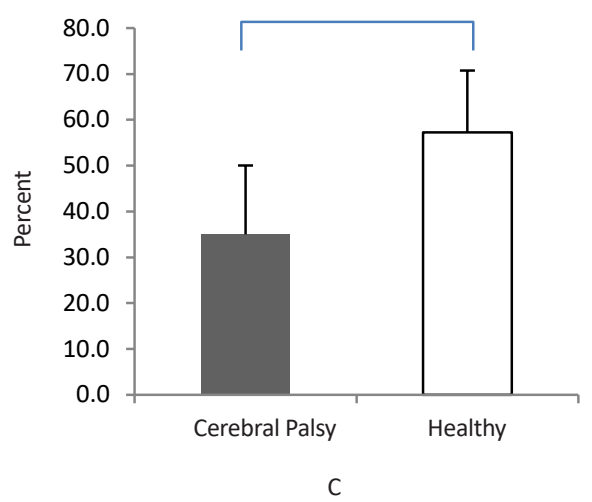

B

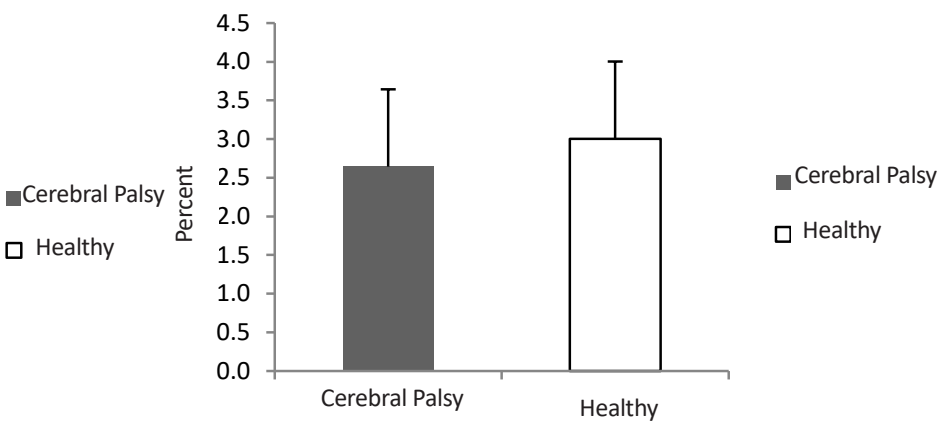

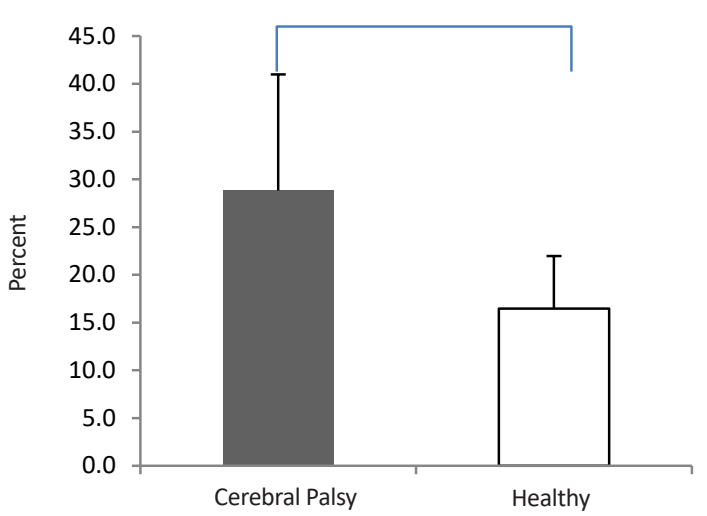

cerebral Palsy

Healthy

PHYSICAL TREA TMENTS

Figure 1. Normalized COM in movement planes (\%)

A. Vertical plane normalized to leg length; B. Anterior/posterior plane normalized to leg length; C. Medial/lateral plane normalized to pelvic width

Various determinants can be used to assess the dynamic balance of children with $\mathrm{CP}$. The most common parameters in this regard are the COM movements range related to the BOS in 3 vertical, anterior/posterior, and medial/lateral directions as well as gait speed $[28,29]$. In the current study, parameters of gait speed, cadence, step length, and motion range of COM of the 3 vertical, anterior/posterior, and medial/lateral directions were employed.

Results suggest that the gait speed and step length of CP children are significantly less than that of normal children. Patients with musculoskeletal disorders reduce walking speed to improve stability. Children with CP increase dynamic balance by slowing the walking speed [4-7] and increasing double support. These results are consistent with other studies that reported walking speed and step length of cerebral palsied children are less than that of healthy children [4-7].

The data of motion range of COM in anterior/posterior directions in both non-normalized and normalized (to leg length) conditions suggest that this variable is significantly lower in children with $\mathrm{CP}$ compared to the healthy subjects, which is in agreement with other studies [5]. Lower movement of COM in anterior/posterior directions indicates that children with $\mathrm{CP}$ cannot move the COM as much as normal children. Toe walking is frequent in these children because of the spasticity of plantar flexor muscles in these children.

Plantar flexor muscles of a normal person with eccentric contraction, store forces in passive muscle tissues

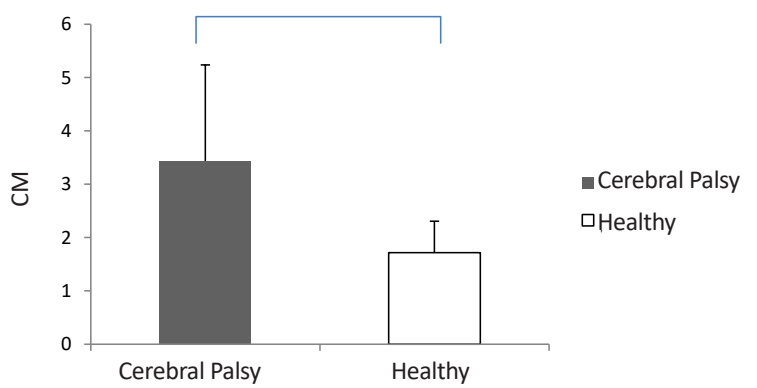

PHYSICAL TREA $\mid$ MENTS

Figure 2. MOS in the $\mathrm{CP}$ and control groups 
and in the plantar fascia during walking (loading rate). Then, during the propulsion phase, with the contraction of these muscles and release of energy stored in the muscles and structures of plantar fascia, one can take a longer step. Because of walking on toes in cerebral palsied children, this normal function of the muscles, especially the ankle muscles and the hip extensors and plantar fascia, is lost and the amount of forward movement decreases [30, 31].

The data of motion range of COM in medial/lateral directions in both non-normalized and normalized (to pelvic width) conditions indicate that this variable in CP children is significantly higher than normal children; which is consistent with other studies [4, 5]. Furthermore, the MOS obtained by subtracting the transverse motion range of COM from the step width, was significantly higher in the $\mathrm{CP}$ children compared to normal ones.

The COM might be outside the BOS in medial/lateral plane which increases the risk of falling in these children. Thus, children with CP use a different walking strategy to avoid falling, and enhance their dynamic balance by increasing the BOS for maintaining the COM inside the BOS in medial/lateral plane. In addition, in the single support phase, increasing the lateral motion of the $\mathrm{COM}$ requires the activity of the hip abductor muscles to resist the gravitational force resulting from the upper body masses [18]. Therefore, because of the inability of these children to produce hip abductor torque for preventing hip and trunk loss on the other side in single support phase, instead of moving the swinging leg in a greater range in the anterior/posterior direction, they move the swinging leg in the lateral direction. Also, by increasing the step width, they try to maintain their balance on the lateral plane [4].

The gait speed, step length, and motion range of the $\mathrm{COM}$ in anterior/posterior and medial/lateral directions are lower in the $\mathrm{CP}$ children. Thus, the reduction of vertical movement of the COM is also plausible. However, our results demonstrate that the motion range of the $\mathrm{COM}$ in vertical direction, both in non-normalized and normalized states, do not differ significantly between the children with and without CP. Maybe the reason for this is that most children with $\mathrm{CP}$ walk on their toes as a result of spasticity in the plantar flexor muscles [23, 24].

They also have increased knee flexion in the dual support phase. Walking on the toes increases the COM. In the dual support phase, in contrast, the COM decreases due to increased knee flexion [32]. Therefore, despite a higher decrease in dynamic balance variables, the COM of children with $\mathrm{CP}$ do not significantly differ from the normal subjects.

The gait speed is an important variable for measuring dynamic balance during walking. By increasing the speed of walking, dynamic balance increases [28, 29]. Reduction in the COM movement in different vertical, anterior/ posterior, and medial/lateral directions when the speed of walking is constant, indicates an increase in the dynamic balance during walking. Increased lateral displacement and decreased anterior/posterior displacement of the COM in CP children indicates a change in their walking pattern. These changes in the COM in various directions are accompanied by slowed walking speed in these children. As a result, the dynamic balance during walking in $\mathrm{CP}$ children decreases. In the present study, because of the inability of $\mathrm{CP}$ children to move on the force plate, it was not possible to record a single step on the force plate. Thus, instead of using the pressure force data, the fifth metatarsal markers data were employed to measure the BOS.

The increase in medial/lateral movement and decrease in anterior/posterior movement of the COM in children with $\mathrm{CP}$ is an indicative of change in their walking pattern. The association between these changes and slowed gait speed suggested a decrease in the dynamic balance of these children during walking.

\section{Ethical Considerations}

\section{Compliance with ethical guidelines}

Ethical aproval was obtained from Ethical Committe of Isfahan University of Medical Sciences.

\section{Funding}

Research Deputy of Isfahan University of Medical Sciences has paid all the costs of the research.

\section{Authors contributions}

Conceptualization and Methodology: All authors; Investigation: Mostafa Kamali, Ali Tahmasebi; WritingOriginal draft: Keyvan Sharifmoradi; Writing-Review and Editing: Mostafa Kamali, Ali Tahmasebi; Funding acquisition: Mostafa Kamali, Ali Tahmasebi; Resources: Keyvan Sharifmoradi; Supervision: All authors.

\section{Conflict of interest}

The authors declared no conflict of interest. 


\section{Acknowledgements}

The authors would like to gratitude Musculoskeletal Research Center and Research Deputy of Isfahan University of Medical Sciences.

\section{References}

[1] Abel MF, Damiano DL. Strategies for increasing walking speed in diplegic Cerebral Palsy. Journal of Pediatric Orthopaedics. 1996; 16(6):753-8. [DOI:10.1097/01241398199611000-00010]

[2] Johnson DC, Damiano DL, Abel MF. The evolution of gait in childhood and adolescent Cerebral Palsy. Journal of Pediatric Orthopaedics. 1997; 17(3):392-6. [DOI:10.1097/01241398199705000-00022] [PMID]

[3] Bruijn SM, Millard M, Van Gestel L, Meyns P, Jonkers I, Desloovere K. Gait stability in children with Cerebral Palsy. Research in Developmental Disabilities. 2013; 34(5):1689-99. [DOI:10.1016/j.ridd.2013.02.011] [PMID] [PMCID]

[4] Kurz MJ, Arpin DJ, Corr B. Differences in the dynamic gait stability of children with Cerebral Palsy and typically developing children. Gait \& Posture. 2012; 36(3):600-4. [DOI:10.1016/j.gaitpost.2012.05.029] [PMID]

[5] Hsue BJ, Miller F, Su FC. The dynamic balance of the children with Cerebral Palsy and typical developing during gait; Part I: Spatial relationship between COM and COP trajectories. Gait \& Posture. 2009; 29(3):465-70. [DOI:10.1016/j. gaitpost.2008.11.007] [PMID]

[6] Hsue BJ, Miller F, Su FC. The dynamic balance of the children with Cerebral Palsy and typical developing during gait; Part II: Instantaneous velocity and acceleration of COM and COP and their relationship. Gait \& Posture. 2009; 29(3):471-6. [DOI:10.1016/j.gaitpost.2008.11.008] [PMID]

[7] Wallard L, Dietrich G, Kerlirzin Y, Bredin J. Balance control in gait children with Cerebral Palsy. Gait \& Posture. 2014; 40(1):43-7. [DOI:10.1016/j.gaitpost.2014.02.009] [PMID]

[8] Jacobson GP, Newman CW, Kartush JM. Handbook of balance function testing. Missouri: Mosby; 1993.

[9] Karimi M, Esrafilian A. Evaluation of the stability of normal subjects and patients with Perthes and spinal cord injury disorders during short and long periods of time. Prosthetics and Orthotics International. 2013; 37(1):22-9. [DOI:10.1177/0309364612446649] [PMID]

[10] Burtner P, Woollacott M, Craft G, Roncesvalles M. The capacity to adapt to changing balance threats: A comparison of children with Cerebral Palsy and typically developing children. Developmental Neurorehabilitation. 2007; 10(3):24960. [DOI:10.1080/17518420701303066] [PMID]

[11] Chen J, Woollacott MH. Lower extremity kinetics for balance control in children with Cerebral Palsy. Journal of Motor Behavior. 2007; 39(4):306-16. [DOI:10.3200/ JMBR.39.4.306-316] [PMID]
[12] Liao HF, Mao PJ, Hwang AW. Test-retest reliability of balance tests in children with Cerebral Palsy. Developmental Medicine \& Child Neurology. 2001; 43(3):180-6. [DOI:10.1017/S0012162201000342]

[13] Rose J, Wolff DR, Jones VK, Bloch DA, Oehlert JW, Gamble JG. Postural balance in children with Cerebral Palsy. Developmental Medicine \& Child Neurology. 2002; 44(01):58-63. [DOI:10.1111/j.1469-8749.2002.tb00260.x]

[14] Ferdjallah M, Harris GF, Smith P, Wertsch JJ. Analysis of postural control synergies during quiet standing in healthy children and children with Cerebral Palsy. Clinical Biomechanics. 2002; 17(3):203-10. [DOI:10.1016/S02680033(01)00121-8]

[15] Owings TM, Pavol MJ, Foley KT, Grabiner MD. Measures of postural stability are not predictors of recovery from large postural disturbances in healthy older adults. Journal of the American Geriatrics Society. 2000; 48(1):42-50. [DOI:10.1111/j.1532-5415.2000.tb03027.x] [PMID]

[16] Karimi MT, Solomonidis S. The relationship between parameters of static and dynamic stability tests. Journal of Research in Medical Sciences. 2011; 16(4):530-5. [PMID] [PMI CID]

[17] Bhatt T, Espy D, Yang F, Pai YC. Dynamic gait stability, clinical correlates, and prognosis of falls among community-dwelling older adults. Archives of Physical Medicine and Rehabilitation. 2011; 92(5):799-805. [DOI:10.1016/j. apmr.2010.12.032] [PMID]

[18] MacKinnon CD, Winter DA. Control of whole body balance in the frontal plane during human walking. Journal of Biomechanics. 1993; 26(6):633-44. [DOI:10.1016/00219290(93)90027-C]

[19] Toebes MJ, Hoozemans MJ, Furrer R, Dekker J, van Dieën $\mathrm{JH}$. Local dynamic stability and variability of gait are associated with fall history in elderly subjects. Gait \& Posture. 2012; 36(3):527-31. [DOI:10.1016/j.gaitpost.2012.05.016] [PMID]

[20] Corriveau H, Hébert R, Raîche M, Dubois MF, Prince F. Postural stability in the elderly: Empirical confirmation of a theoretical model. Archives of Gerontology and Geriatrics. 2004; 39(2):163-77. [DOI:10.1016/j.archger.2004.03.001] [PMID]

[21] Rose J, Gamble JG, Burgos A, Medeiros J, Haskell WL. Energy expenditure index of walking for normal children and for children with Cerebral Palsy. Developmental Medicine \& Child Neurology. 1990; 32(4):333-40. [DOI:10.1111/j.1469-8749.1990.tb16945.x]

[22] Hof A, Gazendam M, Sinke W. The condition for dynamic stability. Journal of Biomechanics. 2005; 38(1):1-8. [DOI:10.1016/j.jbiomech.2004.03.025] [PMID]

[23] Massaad F, Dierick F, Van Den Hecke A, Detrembleur C. Influence of gait pattern on the body's centre of mass displacement in children with Cerebral Palsy. Developmental Medicine \& Child Neurology. 2004; 46(10):674-80. [DOI:10.1017/ S0012162204001136]

[24] Massaad F, Van Den Hecke A, Renders A, Detrembleur C. Influence of equinus treatments on the vertical displacement of the body's centre of mass in children with Cerebral Palsy. Developmental Medicine \& Child Neurology. 2006; 48(10):813-8. [DOI:10.1017/S0012162206001757] 
[25] Dezman ZD, Carollo JJ. Center of mass kinematics during gait initiation in children with Cerebral Palsy. Gait \& Posture. 2006; 24(2):S276-S8. [DOI:10.1016/j.gaitpost.2006.11.189]

[26] Van Den Hecke A, Detrembleur C. Development of displacement of centre of mass during independent walking in children. Developmental Medicine \& Child Neurology. 2004; 46(8):533-9. [PMID]

[27] Beltran EJ, Dingwell JB, Wilken JM. Margins of stability in young adults with traumatic transtibial amputation walking in destabilizing environments. Journal of Biomechanics. 2014； 47(5):1138-43. [DOI:10.1016/j.jbiomech.2013.12.011] [PMID] [PMCID]

[28] Dingwell J, Cusumano J, Sternad D, Cavanagh P. Slower speeds in patients with diabetic neuropathy lead to improved local dynamic stability of continuous overground walking. Journal of Biomechanics. 2000; 33(10):1269-77. [DOI:10.1016/S0021-9290(00)00092-0]

[29] Buzzi UH, Ulrich BD. Dynamic stability of gait cycles as a function of speed and system constraints. Motor Control. 2004; 8(3):241-54. [DOI:10.1123/mcj.8.3.241]

[30] Bennett BC, Abel MF, Wolovick A, Franklin T, Allaire PE, Kerrigan DC. Center of mass movement and energy transfer during walking in children with Cerebral Palsy. Archives of Physical Medicine and Rehabilitation. 2005; 86(11):2189-94. [DOI:10.1016/j.apmr.2005.05.012] [PMID]

[31] Kerrigan DC, Della Croce U, Marciello M, Riley PO. A refined view of the determinants of gait: Significance of heel rise. Archives of Physical Medicine and Rehabilitation. 2000 81(8):1077-80. [DOI:10.1053/apmr.2000.6306] [PMID]

[32] Russell S, Bennett B, Kerrigan D, Abel M. Determinants of gait as applied to children with Cerebral Palsy. Gait \& Posture. 2007; 26(2):295-300. [DOI:10.1016/j.gaitpost.2006.09.079] [PMID] [PMCID] 